\title{
PRINCIPALES VARIABLES PARA LA GESTIÓN DE LA CALIDAD DEL SERVICIO DE RECEPCIÓN DE TURISMO DE CRUCEROS
}

\author{
Sonia Caridad Ruiz Quesada* \\ Universidad de Oriente. Santiago de Cuba \\ Óscar Parada Gutiérrez*** \\ Escuela Superior Politécnica de Chimborazo. Ecuador
}

\section{RESUMEN}

El turismo es una actividad clave en muchos países, constituyendo en la mayoría de las naciones caribeñas la principal fuente generadora de ingresos y de empleo. En particular la modalidad de cruceros constituye una alternativa de desarrollo para los destinos turísticos receptivos del Caribe. Por tal motivo, el objetivo de este artículo es determinar las principales variables que inciden en la gestión de la calidad en la recepción de turismo de cruceros en el destino de Santiago de Cuba. Mediante el análisis de los datos recogidos en entrevistas realizadas a un conjunto de expertos en el período de septiembre a octubre del año 2014 en el destino turístico de Santiago de Cuba, se muestran los resultados obtenidos que permitieron determinar las variables que poseen la mayor incidencia en la calidad del servicio de recepción del turismo de crucero en el destino turístico de Santiago de Cuba. El estudio se realizó aplicando Análisis de Componentes Principales (ACP) a las encuestas a expertos en la materia y se validó el instrumento, a través, del coeficiente Alfa de Cronbach. Posteriormente se sintetizaron los resultados, se hallaron las relaciones entre ellos y, finalmente, se obtuvieron las variables o factores principales que inciden en la calidad del servicio de recepción de cruceros y, ello permitió, el diseño de una metodología para la gestión de la calidad del servicio de recepción de cruceros.

Palabras clave: Turismo de cruceros; destinos turísticos; gestión de la calidad; cruceristas; satisfacción.

Recibido: 10 de febrero de 2015

Devuelto para su revisión: 23 de julio de 2015

Aceptado: 24 de septiembre de 2015

* Departamento de Ciencias Empresariales. Facultad de Ciencias Económicas y Empresariales. Universidad de Oriente. Patricio Lumumba, s/n. SANTIAGO DE CUBA ( Cuba).E-mail: soniac@eco.uo.edu.cu

** Escuela de Ingeniería en Marketing. Facultad de Administración de Empresas. Escuela Superior Politécnica de Chimborazo. Panamericana Sur km. 1 1/2. RIOBAMBA (Ecuador).E-mail: ospg2012@ gmail.com 


\title{
Main Variables for Quality Management of the Reception of Cruise Tourism
}

\begin{abstract}
Tourism is a key activity in many countries, constituting the main source of income and employment in most Caribbean nations. Particularly, cruise modality is an alternative development for receptive tourist destinations in the Caribbean. Therefore, the aim of this article is to identify key variables that affect quality management in the reception of cruise tourism in the destination of Santiago de Cuba. By analyzing the data gathered from interviews with a group of experts in the period from September to October 2014 in the tourism destination of Santiago de Cuba, the results obtained that allowed establishing the appropriate variables with the highest impact on the quality the reception of cruise tourism in the destination of Santiago de Cuba were shown. The analysis was conducted using surveys to experts, Cronbach's Alpha coefficient and Principal Component Analysis (PCA). Through these techniques, the results were synthesized, relations between them were found; and finally, the main variables or factors that led to projecting the design of the methodology for managing the quality of cruise reception were obtained.
\end{abstract}

Keywords: Cruise tourism; tourism destinations; quality management; cruiser; satisfaction.

\section{INTRODUCCIÓN}

\subsection{Generalidades de la investigación}

La calidad ha devenido en estrategia fundamental para la supervivencia de las empresas en el mercado. Los consumidores demandan productos y servicios cada vez más exigentes y, sólo aquellas empresas que logren satisfacer sus expectativas, podrán permanecer operando. El cliente se ha convertido en una fuente de información estratégica sobre la calidad del producto y del servicio, en lugar de ser únicamente el objetivo de las campañas publicitarias de empresas productivas y de servicios. En los destinos turísticos, por ejemplo, se elaboran planes de amplio alcance para satisfacer las necesidades de los clientes. La misión ya no consiste en ser mejores que la competencia, la misión ahora es la excelencia.

En consecuencia, las formas clásicas de dirigir a las organizaciones basadas en el análisis y la optimización de cada una de sus áreas funcionales, van perdiendo vigencia a favor de enfoques de dirección sistémicos que abogan por conseguir un funcionamiento del sistema, suficientemente satisfactorio para cada una de sus partes. La dirección por objetivos, el marketing, la logística y la gestión de la calidad total, entre otros, constituyen formas y campos diferentes, métodos de gestión inspirados en un enfoque integrador y sistémico.

En particular, la gestión de la calidad total es una filosofía de gestión, que promueve la mejora continua (no sólo el aseguramiento de un determinado estándar de calidad, sino su superación permanente) en todas las áreas de la organización (no exclusivamente en el ámbito productivo, como en los enfoques anteriores a ella), actuando fundamentalmente 
sobre las personas, los procesos y orientada, principalmente, a la satisfacción del cliente. En este contexto el sector del turismo se ha convertido en un escenario importante para la aplicación de la gestión de la calidad total. Según refiere la literatura especializada consultada, (Figuerola 1999; Jönsson, C., \& Devonish, D., 2009; Fernández, J. I. P., \& Rivero, M. S., 2010; Ayala H., 2011; OMT, 2013) el turismo es, sin duda, uno de los fenómenos económicos y sociales más importantes de las últimas décadas. Cada año el número de participantes en actividades turísticas aumenta. Como tendencia, en la mayoría de los países del mundo y en especial en los de la región de América Central y el Caribe, el turismo constituye uno de los sectores económicos más dinámicos y con mayor tasa de crecimiento.

Entre las modalidades turísticas conocidas, se encuentra el turismo de cruceros, el cual representa uno de los segmentos del mercado turístico internacional que mayor crecimiento ha experimentado en los últimos años. (Vogel, M., 2011). En particular, la llegada de pasajeros de cruceros al Caribe Insular durante el período 2011-2013 ha tenido un comportamiento muy favorable, con un crecimiento promedio anual de $6,8 \%$, mucho mayor que el crecimiento promedio del Caribe entre los anteriores años (2000-2010), y más alto que el crecimiento medio mundial de las pasajeros que embarcaron en cruceros en el mundo entre el 2011 y el 2013 que, según datos que informa la Cruise Lines Internacional Association (CLIA), se puede calcular en 2,8\%.

El Caribe constituye el primero de los mercados receptores del turismo de crucero, principalmente por el atractivo que representa su clima cálido y soleado, sus espectaculares playas y sus interesantes excursiones. Esta posición como destino de cruceros se debe fundamentalmente a su cercanía respecto al mercado emisor de cruceristas más importante, los Estados Unidos. Según el resumen del desarrollo del crucerismo emitido por Florida-Caribbean Cruise Association (FCCA) a inicios del 2013, el Caribe representa el $40 \%$ de los itinerarios a nivel mundial. De la gran demanda, el $86 \%$ se concentra en 12 grandes empresas que integran la FCCA, que con más de 100 barcos controlan el 94,8\% del mercado norteamericano y el $85,2 \%$ del mercado mundial.

De acuerdo con el «2013 Cruise Industry News Annual Report», el Caribe se mantiene como líder en el negocio mundial de cruceros, pese a un entorno cada vez más competitivo. La estabilidad política, la experiencia de los destinos y la infraestructura creada están entre las fortalezas de la región.

En Cuba reaparece el servicio de recepción de turismo de cruceros en el año 1993, dentro de las estrategias económicas del gobierno cubano para recuperarse de la crisis por la que atravesaba luego del derrumbe del bloque socialista de Europa del Este. Desde esta fecha se ha realizado la recepción de turismo de cruceros en cuatro destinos cubanos: La Habana, Santiago de Cuba, Cienfuegos e Isla de la Juventud, siendo Europa el principal mercado.

La ciudad de Santiago de Cuba, por su infraestructura, es una de las mejores dotadas para la atención a la modalidad turística de crucero en Cuba. Sus condiciones climatológicas, y la posibilidad de ofrecer un clima cálido durante los doce meses del año, así como las características físico geográficas de su bahía, con una privilegiada localización dentro de la isla, ha motivado que prestigiosas compañías de cruceros incursionen en la ciudad de Santiago de Cuba en la modalidad de cruceros (Fred Olsen, NDS Voyage, 
Hapa Lloyd, Costa Cruceros, Pullmantur, entre otras), ofreciendo a Santiago como parte de sus múltiples destinos. Su ubicación y cercanía con las mayores islas antillanas, ha contribuido a la integración exitosa de varias rutas de cruceros en el Caribe, convirtiéndose en un interesante antecedente para el desarrollo y expansión de esta modalidad de turismo. Del mismo modo, el territorio ofrece al crucerismo, además de las condiciones técnicas y operativas, un buen escenario para el disfrute de opciones culturales y otros entretenimientos.

Este estudio tuvo como objetivo determinar las variables que más inciden en la gestión de la calidad del servicio de recepción de cruceros en el destino turístico de Santiago de Cuba. El mismo refiere la no consideración de aquellas variables que, a pesar de incidir en la gestión de la calidad de un destino turístico, no manifiestan relación directa con la gestión del servicio de recepción de cruceros.

\subsection{El contexto de la gestión de la calidad en la recepción del turismo de cruceros en Santiago de Cuba}

El crucerismo reaparece en Cuba durante el año 1993, en un momento donde el turismo era retomado como alternativa económica. Desde la fecha hasta la actualidad, básicamente, se ha operado con el mercado europeo. El mercado norteamericano, no ha podido ser considerado por regulaciones y leyes implantadas por el gobierno de EE.UU.

Entre los años 1995 y el 2005, se dieron importantes pasos en Cuba, acondicionando la infraestructura portuaria, atrayendo importantes compañías europeas, comercializando las operaciones de cruceros en varios destinos de la isla, destacándose La Habana, Santiago de Cuba y Cienfuegos, como puertos y/o puntos de atraque, en conjunto con importantes entidades receptivas de turismo.

El barco de la Compañía italiana Festival se mantuvo realizando escalas hasta el año 2004. Se debe destacar en ese mismo año, el arribo a puerto de la embarcación European Visión de la misma compañía, una de las más grandes de su tipo en aquel entonces con capacidad para 1.890 pasajeros.

Las compañías de cruceros que han operado en Cuba son las siguientes: Pullmantur, Sea Cloud Cruises, Fred Olsen Lines, HapagLloyd Kruezfahrten, Costa Crociere, Delphin Sereisen, Airtours- Sun Cruises, Aída Cruises - Seetours y Costa Line.

Según las estadísticas del Ministerio de Turismo (MINTUR) cubano, en el año 2006, el gasto promedio en tierra por crucerista en el país fue de 158.00 USD por día de escala, de los cuales el $60 \%$ se realizó en tierra; a esto se le debe agregar un promedio de gasto por tripulantes de 72 USD. Cuba se convirtió en puerto madre para la compañía Pullmantur; la embarcación hacía escala en tres puntos alrededor de la isla: La Habana, Cienfuegos e Isla de la Juventud, a la cual, la Compañía, llamó comercialmente «Isla Paraíso».

Durante estos años de operación, las excursiones opcionales a los cruceristas en los diferentes puntos del país han sido bien aceptadas, de tal manera, el 82\% de los cruceristas que visitaron la isla durante el año 2006, realizaron una excursión con un grado de aceptación del $92 \%$.

Estas cifras corroboran que el turismo de cruceros tiene potencial para convertirse en una modalidad altamente rentable para el turismo cubano y uno de los mayores genera- 
dores de ingresos líquidos a corto plazo, considerando que el tiempo de desarrollo de esta modalidad en la isla es corto y se trata de una modalidad poco frecuente, además de que se ha desarrollado básicamente a partir de un mercado marginal.

A partir del año 2007 el pax fee $e^{1}$ que aplican las terminales cubanas se puede considerar alto dentro del Caribe, alcanzando los 15.00 USD por arribo de cruceristas en la Terminal de Cruceros de La Habana y 10.00 en el puerto de Santiago de Cuba. Durante el año 2010, se registró el récord de 102.440 arribos de cruceristas a nivel nacional, cifra que posibilitó que ingresaran al país 1.145.366 USD sólo por concepto de pax fee.

Actualmente se realizan escalas puntuales en diferentes puertos del país. No obstante, embarcaciones de otras compañías europeas como la Black Prince, Delphin, Humbold o la Fred Olsen, continúan realizando escalas en puertos cubanos.

El Ministerio del Turismo (MINTUR) cubano en su planeación estratégica para el 2016 se propone como uno de sus objetivos: «Incrementar el número de operaciones en puertos cubanos». Para ello se preparan como destinos de cruceros los puertos de: La Habana, Cienfuegos, Santiago de Cuba, Holguín, Trinidad, e Isla de la Juventud.

Entre sus principales líneas de acción se encuentran: estimular y priorizar las operaciones lineales con puertos madres en Cuba y que realicen programas de bojeo, potenciar en las ofertas cubanas el empleo de las terminales de crucero de la Habana, Cienfuegos y Santiago de Cuba, contactar con empresas de cruceros que no sean norteamericanas y estimular su entrada a Cuba.

Santiago de Cuba es conocida como la capital del Caribe. La ciudad se encuentra dentro de los principales destinos turísticos de crucero del país, con profundas raíces socioculturales e históricas con altos valores naturales, paisajísticos y climáticos recogidos en una oferta diversa e integral. Por su infraestructura es también una de las mejores dotadas para la atención a la modalidad turística de cruceros. Su ubicación y cercanía con las mayores islas antillanas, han contribuido a la integración exitosa de varias rutas de cruceros en el Caribe, lo cual se convierte en un interesante antecedente para el desarrollo y expansión de esta modalidad de turismo. Con enormes potencialidades, el territorio ofrece para el crucerismo, además de las condiciones técnicas y operativas, un buen escenario para el disfrute de opciones culturales y otros entretenimientos.

Su ubicación geográfica, es considerada privilegiada, ya que se encuentra situada en el paso de importantes rutas marítimas, vinculadas al comercio internacional de nuestra región, principalmente a la transportación de contenedores, así como en el área de un significativo movimiento de cruceros y en un entorno donde están varios puertos de América Central y América del Sur enfrascados en lograr un desarrollo acelerado de su actividad y en franca competencia, que les permita insertarse en el movimiento de mercancías.

Desde el año 1994 hasta octubre del 2013 han arribado a puerto santiaguero 219 buques y 134038 cruceristas, datos que demuestran el interés que tienen compañías de cruceros en el destino Santiago de Cuba.

En correspondencia con las investigaciones desarrollada por los autores de este artículo científico se ha podido constatar que en el destino Santiago de Cuba prevalecen problemas

1 Impuesto aplicado a las embarcaciones por cada persona a bordo en los puertos de destino. 
internos y externos que afectan el desarrollo exitoso de la modalidad de cruceros, identificando los referentes a la gestión de la calidad en la atención al turismo de cruceros como el problema interno más significativo.

Los problemas constatados se pueden resumir en los siguientes:

- Predominio del empirismo para el desarrollo de las operaciones de crucero en el destino turístico.

- Baja calidad en los servicios ofertados al turismo de cruceros.

- Deficiente imagen de la terminal portuaria y su entorno para la atención de cruceros.

- Dificultades en la comercialización del destino.

- Bajo nivel de decisión de la delegación territorial.

- Insuficiente trabajo para potenciar productos tradicionales autóctonos del territorio.

- Pobres conexiones aéreas de la ciudad con otros destinos dentro y fuera del país.

- Falta de integración y apoyo al turismo de los actores de la ciudad.

Existe además un elemento externo que frena significativamente el desarrollo del turismo de crucero en Santiago de Cuba, y es lo referente a la política del gobierno de Estados Unidos, que imposibilita el tránsito de cruceros entre los dos países, debido a regulaciones y leyes implantadas por dicho gobierno. Además de la imposición del bloqueo económico, la llamada Ley Torricelli sanciona a los buques de cualquier nacionalidad que arriben a puertos cubanos a no tocar radas de los Estados Unidos por 180 días: Esto priva a Cuba del tráfico de cruceros en la región, con base en La Florida, al que se le suman las compañías que puedan tener intereses norteamericanos.

Según informe del 2011 de la U.S. Trade Commission, unos 480.000 cruceristas podrían visitar Cuba una vez rectificada la política del gobierno de EE.UU. Estas cifras coinciden con los trabajos y pronósticos elaborados por el MINTUR.

Teniendo en cuenta el comportamiento de los indicadores en la actualidad y el pronóstico antes mencionado se han realizado estimaciones, por las que se espera que si Cuba recibe ese volumen de cruceristas en el año, esto reportaría ingresos por concepto de pax fee alrededor de 7200000 USD, así como ingresos por concepto de gastos de cruceristas en tierra por 36000000 USD, estimación con la que concuerdan autores como Sanders, E., Long, P, (2002). Estas cifras comparadas con los ingresos actuales representarían un aumento de los ingresos en 45 veces.

Los elementos citados anteriormente refieren una importante contradicción a resolver entre el criterio generalizado nacional e internacionalmente, sobre la necesidad de incrementar el nivel de calidad y competitividad de los destinos turísticos de cruceros y la no existencia de un fundamento metodológico y sistémico para la gestión de la calidad del servicio de recepción del turismo de cruceros en Santiago de Cuba.

\subsection{Reflexiones sobre los modelos de gestión de la calidad en los destinos turísticos receptivos de cruceros}

En la determinación de las principales variables que pueden incidir en la calidad del servicio en los destinos turísticos receptivos de cruceros se consideró pertinente tener en 
cuenta la existencia de un conjunto de modelos aplicables al contexto investigado. Esta decisión se fundamentó en una tendencia generalizada en la literatura científica que reconoce que los modelos, además de ser útiles, tienen la capacidad de simplificar situaciones o fenómenos complejos, de modo que revelan lo esencial y sirven de base para la toma de decisiones con carácter proactivo.

En correspondencia con la bibliografía revisada ${ }^{2}$, resultaron significativas las aplicaciones de los siguientes modelos en el contexto de la gestión de la calidad en los destinos turísticos: Modelo SERVQUAL, Modelo SERVPERF, Modelo de Teas, Modelo de Fishbein, Modelo de Calidad de Grouroos - Gummenson, Modelo SERVMAN, Modelo LODGQUAL, Modelo Valper, Sistema Integral de Calidad Turística en Destinos (SICTED), Premio Nacional de Calidad de la República de Cuba, Modelo de Excelencia para la Agencia de Viajes Cubanacan Santiago de Cuba.

Es preciso decir que aún existiendo referencia sobre el tema de la gestión de la calidad en destinos turísticos que reciben turismo de cruceros, no se han encontrado análisis que aborden la gestión de la calidad del servicio de recepción del turismo de cruceros, siendo ésta una de las principales limitaciones teóricas encontradas en la investigación.

El análisis de los modelos refiere que el tema de la calidad se ha orientado, de forma general, a la evaluación y control de la satisfacción del turista sobre los factores que resultan determinantes en ello y en los atributos tangibles del producto turístico. Del mismo modo los aspectos menos trabajados son: la inclusión de las dimensiones técnicas del servicio en las mediciones y la propuesta de procedimientos de mejora así como enfoques globales que no consideran las especificidades de las modalidades turísticas en un destino particular; tal es el caso del turismo de cruceros.

Por medio del análisis de los modelos referenciados en este epígrafe, se pudo arribar a conclusiones desde dos puntos de vista; por una parte, la identificación de elementos comunes que sirven de base teórica para la propuesta de esta investigación, así como elementos que limitan la pertinencia de los modelos estudiados para lograr la gestión de la calidad del servicio de recepción de turismo de cruceros en el contexto del destino Santiago de Cuba.

Elementos comunes:

- Se estructuran en factores o etapas, interrelacionados.

- Refieren la necesidad de una gestión integrada.

- Contribuyen al incremento de la competitividad del producto turístico.

- Promueven de modo directo o indirecto el desarrollo sostenible.

- Declaran la necesidad de la aplicación de herramientas para el control de la gestión turística.

- La satisfacción de la demanda turística es un factor clave para alcanzar la eficiencia económica de la actividad turística.

2 Cronin, J. J., \& Taylor, S. A. 1994; Getty, J.; Thompson, K. 1994; Dachary, A. C., \& Arnaiz Burne, S. M. 2006; Martínez, E. O., et. al 2008; Mock Díaz J, 2007; Robles, C. F., \& López, A. M. G. 2009; Barbosa, A. J., \& Gutiérrez, O. P. 2010; Vergara, J. C., Quesada, V. M., \& Blanco, I. 2011; Ferreira Lopes, S. D., \& Veloso da Silva, J. A. 2011; Mora Contreras, C. E. 2011; Sánchez Mendoza, V. V. 2012; Premio Nacional de Calidad de la República de Cuba, 2013; Plan estratégico de desarrollo del destino turístico de Santiago de Cuba, 2013. 
Principales limitaciones de los modelos estudiados:

- Están orientados en general a la evaluación de la calidad del servicio, teniendo en cuenta la satisfacción del cliente.

- Aunque son útiles para el diagnóstico, al detectar qué anda mal, no ofrecen todas las herramientas y métodos necesarios para determinar las causas y vías de mejoras.

- No se desarrolla acertadamente el enfoque por procesos.

- Carácter reactivo e incapacidad para determinar los factores que limitan la obtención de resultados superiores.

- Es común que muestren más el punto de vista de los directivos de las organizaciones y de los investigadores, que de los propios clientes, al preestablecer los atributos y requisitos que se deben considerar, de aquí que no se tenga en cuenta la importancia relativa de los atributos y requisitos según el criterio de los clientes.

- Ninguno de los modelos valorados ha orientado su aplicación a un destino turístico de cruceros.

En ninguna de las referencias especializadas consultadas se trata el tema de la gestión de la calidad en la atención al turismo de cruceros. Si bien el análisis de los enfoques de calidad permitió conocer los puntos comunes entre éstos y hacia dónde debe apuntar cualquier nueva propuesta o, al menos, qué no podría dejar de considerar ésta; también corroboró que los enfoques existentes de gestión de la calidad, no resultan suficientes para enfrentar los retos que se imponen para los destinos turísticos de cruceros, sobre todo en lo referido a la diversidad de procesos que incluye, el escaso nivel de integración y calidad en la ejecución de procesos, que impactan en la satisfacción del turismo de cruceros, la no existencia de una estructura de dirección integradora del conjunto de actores que operan en el destino turístico para la atención a cruceros y la no definición con precisión de las fronteras del sistema que conforman el destino de cruceros.

Del análisis de estos modelos se constató la presencia de 39 variables que tienen alta incidencia en la calidad. En el Anexo 1 inciso a, se muestra una tabla en la que aparece la relación de variables por modelos. Dichas variables inciden en la gestión integrada y de calidad de destinos turísticos pero su intensidad varía de acuerdo a su contexto particular. Las variables que se incluirán en el estudio y una breve explicación de las mismas aparecen relacionadas en el Anexo 1 inciso b.

\section{METODOLOGÍA}

En la investigación se aplicó el coeficiente Alpha de Cronbach para validar la confiabilidad y validez del instrumento de medición y el Análisis de Componentes Principales (ACP) para reducir las dimensiones del conjunto de variables.

Los datos utilizados para este estudio fueron resultado de la encuesta aplicada a 50 expertos del destino turístico Santiago de Cuba, implicando tanto a gerentes, como personal de contacto directo en la atención a los cruceristas. En esta composición participaron representantes de la dirección del destino turístico, de agencias de viajes que realizan la 
contratación de cruceros, representantes de los turoperadores (TT.OO) en el destino, guías de turismo y funcionarios del gobierno local que están vinculados al servicio analizado.

Los criterios de selección de los expertos para este estudio fueron el nivel de conocimiento, experiencia y estudios realizados, que determinan el cálculo de un coeficiente de competencia.

La competencia de expertos se determinó por el coeficiente K, el cual se calculó de acuerdo con la opinión del experto sobre su nivel de conocimiento acerca del problema que se está analizando y con las fuentes que permitan argumentar sus criterios, para ello se emplea la relación 1.

$$
\mathrm{K}=1 / 2(\mathrm{Kc}+\mathrm{Ka})
$$

Donde:

Kc: Es el coeficiente de conocimiento e información que tiene el experto acerca del tema investigado. Para ello se evalúa una lista de criterios que refieren una relación esencial entre el sujeto (experto) y el objeto. Se destaca la creatividad, la experiencia, conocimiento, capacidad de análisis, intuición.

Ka: Es el coeficiente de argumentación o fundamentación de los criterios del experto para evaluar el nivel de influencia de cada una de las fuentes de sus criterios (estudios realizados en el país o en el extranjero, publicaciones de alto impacto, evidencias de cursos realizados y aprobados, resultados de la evaluación del desempeño en los últimos cinco años, entre otros).

Para esta investigación se utilizó la opción Evaluación de Expertos del software DECICIÓN ${ }^{3}$. La aplicación de este software confirmó que del total de los posibles expertos (75), 50 resultaron competentes para tomar en cuenta sus valoraciones en esta investigación.

Los 50 expertos asignaron valores a cada una de las variables analizadas según su valoración acerca de la incidencia que presentaban en la gestión de la calidad del servicio de recepción de cruceros en Santiago de Cuba.

Para medir el grado de incidencia de cada una de las variables en la gestión del servicio estudiado se estableció una escala tipo Likert de 1 a 5 donde 1 es el menor grado de incidencia y 5 el mayor, tal y como se muestra en el formato de la Tabla 1.

\section{Tabla 1}

ESCALA DE EVALUACIÓN DE LA INCIDENCIA DE LAS VARIABLES EN LA GESTIÓN DE LA CALIDAD DEL SERVICIO DE RECEPCIÓN DE CRUCEROS

\begin{tabular}{|c|c|c|c|c|}
\hline $\begin{array}{c}\text { Incidencia } \\
\text { Nula }\end{array}$ & $\begin{array}{c}\text { Incidencia } \\
\text { Baja }\end{array}$ & $\begin{array}{c}\text { Incidencia } \\
\text { Media }\end{array}$ & $\begin{array}{c}\text { Incidencia } \\
\text { Alta }\end{array}$ & $\begin{array}{c}\text { Incidencia } \\
\text { Muy Alta }\end{array}$ \\
\hline 1 & 2 & 3 & 4 & 5 \\
\hline
\end{tabular}

Fuente: elaboración propia.

\footnotetext{
3 Desarrollo informático de apoyo a la toma de decisiones grupales creado en la Universidad de Matanzas, Cuba.
} 
Una vez recogida las valoraciones de todos los expertos se pasó a validar la concordancia entre ellos, se utilizó para ello la prueba W de Kendall. Como soporte informático se utiliza el paquete estadístico SPSS ${ }^{4}$ Versión 19.0. Las hipótesis a evaluar fueron las siguientes:

Prueba de hipótesis H1: El juicio de los expertos es consistente.

H0: El juicio de los expertos no es consistente.

En la Tabla 2 se muestra el resultado obtenido.

Tabla 2

ESTADÍSTICOS DE CONTRASTE

\begin{tabular}{|l|c|}
\hline N & 50 \\
W de Kendalla &, 811 \\
Chi-cuadrado & 1054,260 \\
Gl & 26 \\
Sig. asintót. &, 000 \\
\hline
\end{tabular}

a Coeficiente de concordancia de Kendall. Fuente: Software SPSS.

Según los resultados de la Tabla 2 se corroboró la existencia de una alta concordancia en el criterio emitido por los expertos, con un valor del coeficiente W de Kendall de 0.811 y una alta significación, menor que 0.05 , que permite no rechazar la hipótesis H1.

\section{ANÁLISIS Y DISCUSIÓN DE LOS RESULTADOS}

Para comenzar con la selección de las 39 variables se siguieron los siguientes pasos:

En un primer momento del análisis, se seleccionaron aquellas variables a las cuales todos los expertos otorgaron la misma calificación; además en estas variables el criterio del total de expertos coincidió con que presentan una «Muy Alta» y «Alta Incidencia» en la gestión de la calidad del servicio de recepción del turismo de cruceros en un destino turístico receptivo. Ellas fueron: cliente, medio ambiente, perfeccionamiento, objetivos, organización, producto o servicio, recursos materiales, proveedores de servicios turísticos, tecnología, valores, visión, retroalimentación, implicados en el servicio, fidelización y satisfacción.

Una vez seleccionadas estas 15 variables como de «Muy Alta» y «Alta Incidencia», se decidió clasificarlas dentro del grupo de variables a tener en consideración para el estudio de la gestión del servicio de recepción de cruceros.

4 SPSS. Statistical Paquet for Social Science. IBM. 
Posteriormente, en un segundo momento, con las restantes 24 variables se aplicó el Coeficiente Alpha de Cronbach para evaluar la confiabilidad y validez lograda con el instrumento de medición. Este coeficiente plantea que si hay varios sujetos que responden a los ítems (variables), entonces se puede calcular la varianza para cada variable y la varianza para la suma. La varianza de la suma será más pequeña que la suma de las varianzas de las variables, si las variables miden la misma variabilidad entre sujetos, es decir, si ellos miden el mismo score. Técnicamente, la varianza de la suma de dos variables es igual a la suma de las varianzas menos (dos veces) la covarianza, es decir, la cantidad verdadera de variación común a las dos variables.

Se puede, por tanto, estimar la proporción de la verdadera variación que es capturada por las variables, comparando la suma de las variaciones de las variables con la variación de la suma. Específicamente, se puede calcular:

$$
\mathrm{a}=(\mathrm{k} /(\mathrm{k}-1)) *\left[1-\mathrm{S}\left(\mathrm{s}_{\mathrm{i}}{ }_{\mathrm{i}}\right) / \mathrm{s}^{2}{ }_{\text {sum }}\right]^{5}
$$

La relación (2) es la fórmula para el índice más común de fiabilidad, que la literatura especializada reconoce como Coeficiente Alfa de Cronbach.

Si la característica medida no es verdadera, sino que sólo hay errores en las variables (el cual es esotérico y único, por consiguiente, incorrelacionado entre los sujetos), entonces la varianza de la suma será igual que la suma de las varianzas de las variables individuales; por consiguiente, el coeficiente Alfa será igual a cero. Si todas las variables son absolutamente confiables y miden la verdadera característica, entonces el Coeficiente Alfa es igual a 1 .

La confiabilidad de la escala se calcula mediante diversos métodos pero en la mayoría de ellos la escala de evaluación oscila entre valores de 0 y 1 , donde 0 significa nula confiabilidad y 1 máxima confiabilidad.

Tabla 3

ESCALA DE EVALUACIÓN DEL COEFICIENTE ALFA DE CRONBACH

\begin{tabular}{|c|c|c|c|c|}
\hline Baja confiabilidad & Confiabilidad media & Regular & Aceptable & Elevada \\
\hline 0.25 & 0.50 & 0.75 & 0.90 & 1.00 \\
\hline
\end{tabular}

Fuente: elaboración propia a partir de Hair, J. F., Sarstedt, M., Ringle, C. M., \& Mena, J. A. (2012).

Con el apoyo del Software STATISTICA y en particular la opción de Técnicas Exploratorias Multivariadas \Análisis de confiabilidad de los ítems, se calcularon las medias y desviaciones típicas para cada variable como se muestra en la Tabla 4, así como el coeficiente Alpha resultante, una vez eliminada una variable (Alpha if deleted). A continuación se muestra un ejemplo de este análisis.

5 Lizasoain, L; Joaristi. L. (2003). Gestión y análisis de datos con SPSS versión 11. Universidad del País Vasco. Ed. Thomson-Paraninfo. Madrid. 
Tabla 4

ANÁLISIS DE CONFIABLIDAD DE LAS VARIABLES

\begin{tabular}{|c|l|l|l|l|}
\hline $\begin{array}{c}\text { No. } \\
\text { Variable }\end{array}$ & Nombre de la Variable & Media & $\begin{array}{c}\text { Desviación } \\
\text { Típica }\end{array}$ & $\begin{array}{c}\text { Alpha if } \\
\text { deleted }\end{array}$ \\
\hline 1 & Acciones preventivas & 4.942857 & 0.235504 & 0.880495 \\
\hline 2 & Auditoría & 1.942857 & 0.235504 & 0.880495 \\
\hline 3 & Aseguramiento metrológico & 1.057143 & 0.235504 & 0.919444 \\
\hline 4 & Comunicación & 4.942857 & 0.235504 & 0.880495 \\
\hline 5 & Indicadores & 4.942857 & 0.235504 & 0.880495 \\
\hline 6 & Costo & 4.942857 & 0.235504 & 0.880495 \\
\hline 7 & Documentos & 3.942857 & 0.235504 & 0.880495 \\
\hline 8 & Actividades-operaciones & 4.942857 & 0.235504 & 0.880495 \\
\hline 9 & Inspección & 2.057143 & 0.235504 & 0.919444 \\
\hline 10 & Liderazgo & 3.057143 & 0.235504 & 0.919444 \\
\hline 11 & Comercialización & 4.942857 & 0.235504 & 0.880495 \\
\hline 12 & Motivación & 4.028571 & 0.169031 & 0.908319 \\
\hline 13 & Patentes & 1.057143 & 0.235504 & 0.919444 \\
\hline 14 & Planeación & 4.942857 & 0.235504 & 0.880495 \\
\hline 15 & Política & 3.942857 & 0.235504 & 0.880495 \\
\hline 16 & Personal & 2.942857 & 0.235504 & 0.880495 \\
\hline 17 & Principios & 2.942857 & 0.235504 & 0.880495 \\
\hline 18 & Registros de calidad & 1.942857 & 0.235504 & 0.880495 \\
\hline 19 & Selección & 4.942857 & 0.235504 & 0.880495 \\
\hline 20 & Servicios de postventa & 1.057143 & 0.235504 & 0.919444 \\
\hline 21 & Seguridad e higiene & 3.971429 & 0.169031 & 0.899693 \\
\hline 22 & Sistema informativo & 4.942857 & 0.235504 & 0.880495 \\
\hline 23 & Sociedad & 0.235504 & 0.880495 \\
\hline 24 & Técnicas estadísticas & 0.235504 & 0.880495 \\
\hline
\end{tabular}

Fuente: Software STATISTICA.

Los resultados mostrados en la Tabla 4 refieren que existen cinco variables que sus medias son menores que dos, o sea que presentan incidencia nula en la gestión de la calidad del servicio de recepción a cruceros. Por otra parte, cuando se analizan los resultados del coeficiente Alpha de Cronbach, se observa que su exclusión del análisis mejora el valor de dicho coeficiente. Dichas variables son: auditoría, aseguramiento metrológico, patentes, registros de calidad y servicios de postventa.

Al excluir estas variables, se repite el análisis y el coeficiente estandarizado que se obtiene muestra un valor de 0,937075 , indicando que la confiabilidad es elevada. Se observa además que la variable que eleva el coeficiente y además su media al valor de 2,05 es la 
variable «Inspección», a la que los expertos calificaron con una incidencia débil y, por tanto, se excluye también del estudio. Este análisis se realiza por tercera vez quedando el coeficiente Alfa de Cronbach calculado con valor de 0,956322, el cual es elevado. Por tanto, todas las variables resultantes del estudio poseen incidencia de «Media» a «Muy Alta». De este modo quedaron 33 variables que presentan influencia en la gestión de la calidad del servicio de recepción del turismo de crucero en el destino receptivo. Posteriormente se listan las variables excluyendo las eliminadas por el análisis del coeficiente Alpha de Cronbach.

\subsection{Análisis de Componentes Principales}

Una vez determinadas estas 33 variables se desea reducir las dimensiones de este conjunto y para ello se aplicó el Análisis de Componentes Principales (ACP), ya que esta técnica permite el tratamiento conjunto de las variables observadas, reduciendo así el número de datos y consiguiendo identificar un grupo de variables ficticias formadas a partir de la combinación de las anteriores observadas. De esta forma, se sintetizan los datos y se relacionan entre sí, sin hacer ninguna hipótesis previa sobre lo que significa cada factor inicial.

Las macrocaracterísticas o componentes principales, que se obtienen tras un proceso de cálculo de raíces y vectores característicos de una matriz simétrica, tienen como objetivo contener la mayoría de la varianza observada, con lo que se evita conseguir información redundante. Para que esto suceda, las variables han de ser incorrelacionadas entre sí y se han de poder expresar como combinación lineal de las variables que realmente han sido observadas. A mayor varianza incorporada en cada una de estas componentes, implica que la misma contiene una mayor cantidad de información.

Se ha realizado una aproximación ${ }^{6}$ a los factores que podrían ser considerados principales en la gestión del servicio de recepción del turismo de crucero en el destino Santiago de Cuba. Utilizando esta técnica y aplicándola a las respuestas obtenidas sobre la valoración de las variables (33 variables determinadas a través del análisis del coeficiente Alpha de Cronbach), se intenta conocer, a su vez, si éstas se pueden concretar y reducir a un número menor, sin perder demasiada información, resumiendo de esta forma las que presentan un peso específico mayor y evitando redundancias que puedan estar contenidas en más de una variable. Después de un análisis descriptivo inicial, se obtuvieron los estadísticos, considerando finalmente un total de 33 variables para incorporar al modelo.

\section{Tabla 5}

\section{KMO Y PRUEBA DE BARTLETT}

\begin{tabular}{|l|l|r|}
\hline Medida de adecuación muestral de Kaiser-Meyer-Olkin. &, 891 \\
\hline Prueba de esfericidad de Bartlett & Chi-cuadrado aproximado & $6.791,740$ \\
\hline & gl & 178 \\
\hline & Sig. &, 000 \\
\hline
\end{tabular}

Fuente. Salida del software SPSS.

6 Se utilizó el Software estadístico incorporado en el paquete SPSS 15.0. 
La Tabla 5 refiere los estadísticos correspondientes al estudio de la adecuación de la muestra al modelo. Se pudo comprobar que la adecuación muestral para este análisis no sólo resulta deseable, sino que presenta una buena adecuación a los datos, ya que el estadístico de KMO, que indica la proporción de la varianza que tienen en común las variables analizadas, presenta un valor de 0,891 , coeficiente muy cercano a la unidad, que es lo más aconsejado $^{7}$ para este tipo de análisis, lo que indica una perfecta adecuación de los datos a un modelo de análisis factorial. Mediante la prueba de esfericidad de Bartlett, se asegura que, si el nivel crítico es superior a 0,05 , entonces no se podrá rechazar la hipótesis nula de esfericidad. Como se comprobó en el análisis, la significación es casi perfecta, ya que obtiene el valor 0,000 , menor que 0,05 , por lo que se puede rechazar la hipótesis nula, considerando el ajuste de las variables mediante el análisis factorial idóneo.

Otro elemento importante que permite comprobar la condición necesaria para continuar con la metodología de ACP es que, una vez introducidas las variables, los datos obtenidos ofrezcan una matriz definida positiva, lo que ocurre en este estudio.

Para este análisis se calcula el coeficiente de correlación parcial, el cual indica la relación existente entre dos variables si se elimina el efecto del resto de variables del modelo. En la Tabla 6 se muestra un extracto de la matriz de correlaciones, la cual presenta en la diagonal principal valor de 1 , que expresa la correlación perfecta y valores críticos cercanos a cero en caso de ser incorrelacionadas.

Tabla 6

EXTRACTO DE LA MATRIZ DE CORRELACIONES a,b

\begin{tabular}{|l|r|r|r|r|r|r|r|r|}
\hline & $\begin{array}{c}\text { Acciones } \\
\mathrm{P}\end{array}$ & Clientes & Comunicación & Indicadores & Costo & Documentos & $\ldots$ & Satisfacción \\
\hline Acciones P & 1.000 & .167 & .417 & .471 & .750 & .091 & $\ldots$ & .479 \\
\hline Clientes & .502 & 1.000 & .167 & .354 & .417 & .730 & $\ldots$ & .404 \\
\hline Comunicación & .453 & .167 & 1.000 & .471 & .167 & .091 & $\ldots$ & .303 \\
\hline Indicadores & .629 & .354 & .471 & 1.000 & .354 & .258 & $\ldots$ & .107 \\
\hline Costo & .308 & .417 & .167 & .354 & 1.000 & .091 & $\ldots$ & .404 \\
\hline Documentos & .313 & .730 & .091 & .258 & .091 & 1.000 & $\ldots$ & .359 \\
\hline $\begin{array}{l}\text { Actividades- } \\
\text { operaciones }\end{array}$ & .051 & .548 & .091 & .258 & .548 & .400 & $\ldots$ & .608 \\
\hline Liderazgo & .262 & .697 & .253 & .299 & .106 & .509 & $\ldots$ & .211 \\
\hline $\begin{array}{l}\text { Medio } \\
\text { Ambiente }\end{array}$ & .000 & .167 & .067 & .519 & .300 & .292 & $\ldots$ & .373 \\
\hline$\ldots$ & .228 & .513 & .303 & .107 & .404 & .359 & $\ldots$ & $\ldots$ \\
\hline Satisfacción & & & $\ldots$ & $\ldots$ & $\ldots$ & $\ldots$ & $\ldots$ \\
\hline
\end{tabular}

Fuente: Software SPSS. a. Determinante $=$ a 8.70 E-019 b. Esta matriz es definida positiva.

7 A partir de 0,5 se considera que es buena la adecuación muestral para un análisis factorial. 


\subsection{Extracción de factores}

Las denominadas comunalidades en el inicio de una técnica de ACP siempre son iguales a uno, cobrando sentido en los componentes obtenidos al final. Al considerar tan sólo un número reducido de factores entre todas las variables observadas, la varianza total no queda totalmente explicada. Por tanto, sus valores oscilarán entre cero y uno, es decir, entre la posibilidad de que los factores comunes no expliquen nada de la variabilidad de una variable o que, por el contrario, ésta quede totalmente explicada por los factores comunes.

En la Tabla 7 se presentan las comunalidades obtenidas para cada una de las variables, una vez realizada la extracción. Así, se puede comprobar que variables tales como el cliente con un 0,942 de comunalidad, indicadores con un 0,891 o productos y/o servicios con un 0,882 , explican en mayor proporción la varianza según su participación en los factores o componentes resultantes en el análisis, mientras que variables como documentos y sistemas informáticos participan en menor medida en la explicación de dicha varianza.

Tabla 7

EXTRACTO DE LA MATRIZ DE COMUNALIDADES

\begin{tabular}{|l|r|r|}
\hline Variables & Inicial & Extracción \\
\hline Acciones preventivas & 1.000 & .784 \\
Cliente & 1.000 & .942 \\
Comunicación & 1.000 & .804 \\
Indicadores & 1.000 & .891 \\
Costo & 1.000 & .849 \\
Documentos & 1.000 & .616 \\
Actividades-operaciones & 1.000 & .831 \\
Liderazgo & 1.000 & .723 \\
Medio ambiente & 1.000 & .852 \\
Seguridad e higiene & 1.000 & .796 \\
Sistema informativo & 1.000 & .694 \\
Sociedad & 1.000 & .810 \\
Tecnología & 1.000 & .879 \\
Técnicas estadísticas & 1.000 & .778 \\
Valores & 1.000 & .851 \\
Visión & 1.000 & .879 \\
Retroalimentación & 1.000 & .740 \\
Implicados en el servicio & 1.000 & .801 \\
Fidelización & 1.000 & .811 \\
..... & $\ldots .$. & $\ldots .$. \\
Satisfacción & 1.000 & .736 \\
\hline Fuent software SPSs & &
\end{tabular}

Fuente: software SPSS. 
El proceso matemático desarrollado por Harman, (1980) por el que se desarrolla la elección de las componentes principales de una muestra, se realiza a partir de una matriz de correlaciones, de la cual, a través de la aplicación del análisis factorial correspondiente, se extrae otra que se denomina factorial. Las columnas de esta última matriz representan a un factor y las filas coinciden en número con el total de variables observadas. A cada elemento de esta matriz factorial se le denomina peso, carga, ponderación o saturación factorial y son interpretados como índices de correlación entre las filas y las columnas, indicando así el peso que cada variable asigna a cada factor.

Al analizar la Tabla 8 se puede decir que para el análisis se extraerán 7 componentes o factores principales. En la bibliografía consultada se recogen las reglas para saber el número más adecuado a conservar, por ejemplo, la que se conoce como Criterio de Kaiser, que indica que hay que conservar los componentes principales cuyos valores propios son mayores que la unidad, aunque el criterio más utilizado es el de observar el porcentaje de varianza total explicada por cada componente o factor y, cuando éste llega a un porcentaje acumulado considerado alto normalmente cerca del $80 \%$, significa que el número de factores es suficiente.

En los resultados mostrados en la Tabla 8 se observa que, a partir de la componente número siete el autovalor comienza a ser inferior a la unidad y, además, el porcentaje de la varianza explicada acumulada asciende al $82,896 \%$, valor que se considera lo suficientemente alto para estimar que siete es un número de factores suficiente.

Una vez definido el número de componentes a extraer, se obtienen las matrices de componentes y de componentes rotados que se presentan en las Tablas 9 y 10 respectivamente. Estas tablas indican las coordenadas de cada variable en cada componente y de ahí la inclusión de las mismas de una forma u otra en cada componente.

El procedimiento que transforma la matriz factorial inicial (Tabla 9) en otra denominada matriz factorial rotada (Tabla 10), más fácil de interpretar, consiste en una combinación lineal de la primera, que explica la misma cantidad de varianza inicial. Los factores rotados tratan de que cada una de las variables originales tenga una correlación lo más próxima a uno que sea posible, con uno de los factores y correlaciones próximas a cero con los restantes, consiguiendo así correlaciones altas con un grupo de variables y bajas con el resto.

Para poder realizar una interpretación más sencilla, se utiliza el sistema de rotación de los factores empleado la Normalización Varimax, convergiendo dicha rotación en 11 iteraciones, indicando que se realizará la extracción para siete componentes ya que, como se explicó anteriormente, con este número de componentes se consigue obtener más del $80 \%$ de la varianza explicada.

A continuación se presenta la matriz de componentes rotados, mostrando, en este caso, sólo los valores de las coordenadas mayores para cada variable, según el componente en el que se ubican. 


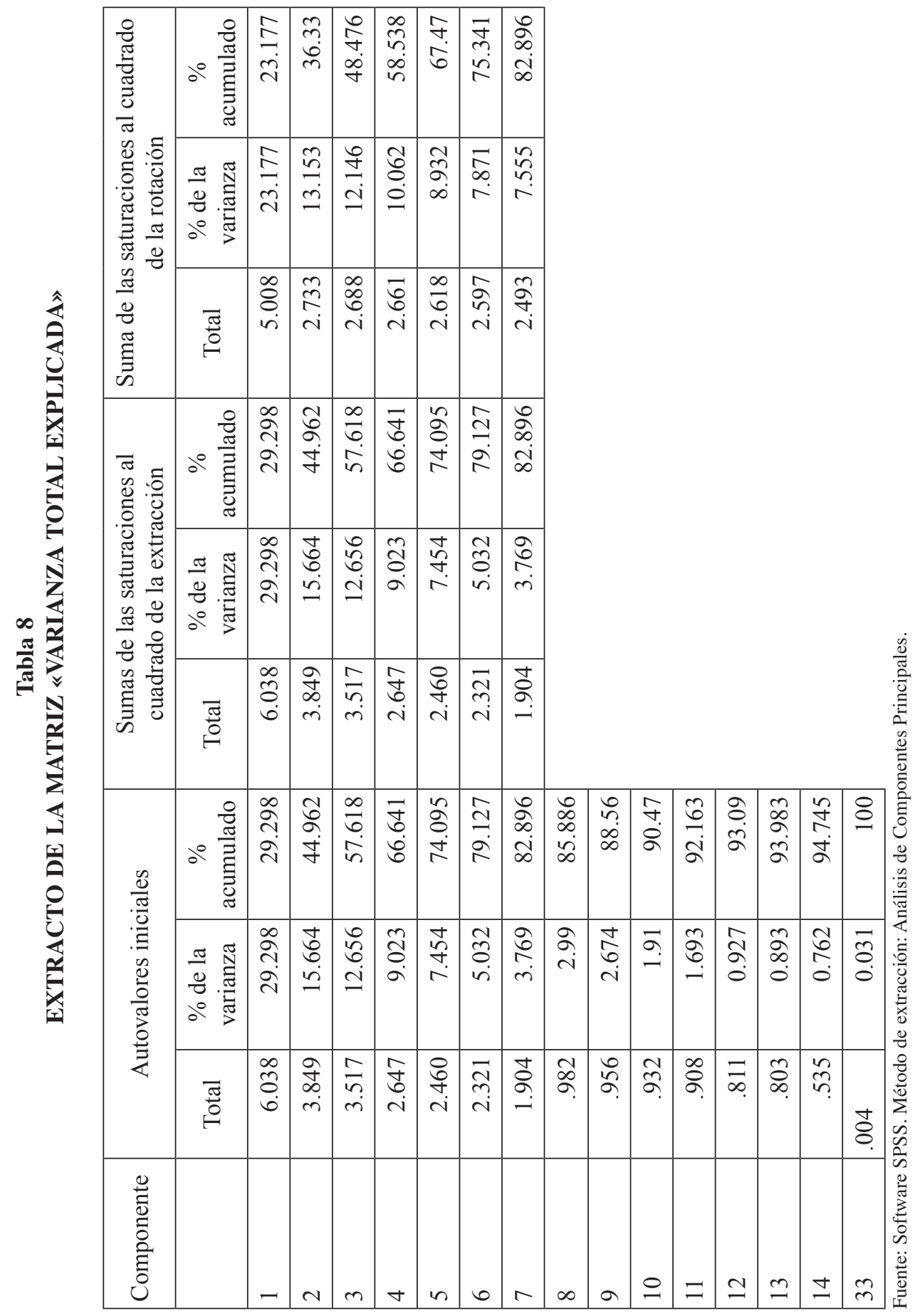


Tabla 9

EXTRACTO DE LA MATRIZ DE COMPONENTES (a)

\begin{tabular}{|c|c|c|c|c|c|c|c|}
\hline & \multicolumn{7}{|c|}{ Componente } \\
\hline & 1 & 2 & 3 & 4 & 5 & 6 & 7 \\
\hline Proveedores de servicios turísticos & .760 & .202 & .099 & .041 & .229 & .006 & .304 \\
\hline Fidelización & .760 & .224 & .011 & .076 & .352 & .155 & .044 \\
\hline Principios & 679 & .165 & .001 & .198 & .076 & .026 & .360 \\
\hline Satisfacción & 655 & .476 & .117 & .020 & .088 & .014 & .169 \\
\hline Medio ambiente & .625 & .280 & .029 & .251 & .367 & .150 & .243 \\
\hline Tecnología & .573 & .015 & .192 & .024 & .558 & .065 & .327 \\
\hline Personal & .562 & .005 & .146 & .308 & .257 & .148 & .282 \\
\hline Valores & .532 & .260 & .001 & .376 & .225 & .009 & .510 \\
\hline Selección & .436 & .083 & .225 & .416 & .016 & .077 & .056 \\
\hline Cliente & .358 & .287 & .343 & .029 & .349 & .144 & .218 \\
\hline Indicadores & .282 & .713 & .357 & .149 & .272 & .135 & .237 \\
\hline Sistema informativo & .229 & .591 & .434 & .051 & .027 & .001 & .066 \\
\hline Acciones preventivas & .037 & .591 & .278 & .056 & .228 & .446 & .244 \\
\hline Comercialización & .233 & .579 & .217 & .119 & .028 & .327 & .215 \\
\hline Retroalimentación & .548 & .558 & .061 & .042 & .179 & .065 & .134 \\
\hline Liderazgo & .449 & .491 & .068 & .347 & .023 & .153 & .128 \\
\hline Perfeccionamiento & .064 & .086 & .470 & .173 & .116 & .584 & .156 \\
\hline Costo & .288 & .054 & .448 & .413 & .355 & .501 & .016 \\
\hline Seguridad e higiene & .110 & .461 & .059 & .274 & .187 & .141 & .522 \\
\hline$\cdots \cdots$ & $\ldots$ & $\cdots$ & $\cdots$ & $\cdots$ & $\cdots$ & $\cdots$ & $\cdots \cdots$ \\
\hline Organización & .270 & .373 & .035 & .146 & .083 & .301 & .450 \\
\hline
\end{tabular}

Fuente: Software SPSS. Método de extracción: Análisis de componentes principales.

(a) 7 componentes extraídos 
Tabla 10

MATRIZ DE COMPONENTES ROTADOS MODIFICADA

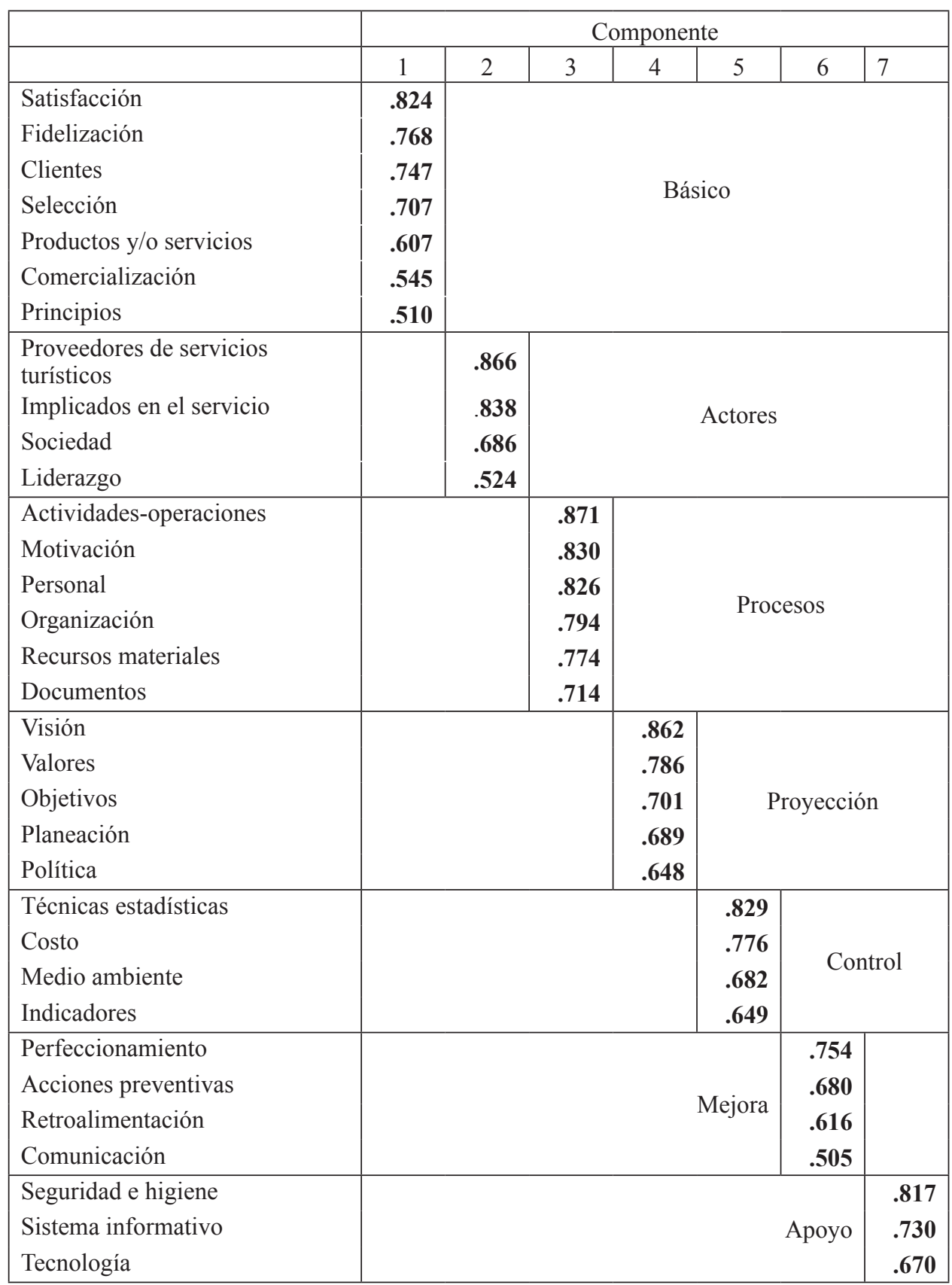

Fuente: adaptado a partir de los resultados del Software SPSS. 
A continuación se renombran los componentes en función de las variables que incorporan.

Componente 1. En este primer componente se ubican siete variables, ellas son: satisfacción, fidelización, clientes, selección, productos y/o servicios, comercialización y principios. Al analizar estas variables y la implicación en la gestión de la calidad del servicio estudiado, se propone denominar este componente como «Factor básico», este factor explica por sí solo el 29,29\% de la varianza total, ocupando un lugar destacado frente al resto de los componentes.

Componente 2. Contiene cuatro variables y explica el 15,66\% de la varianza total, a este componente se le llamará «Factor Actores», ya que son variables relacionadas con los proveedores de servicios, implicados en el servicio de recepción de cruceros, liderazgo y la sociedad.

Componente 3. Incluye seis variables que describen elementos fundamentales del enfoque en proceso, como: actividades-operaciones, recursos materiales, personal, documentos, organización y motivación. Por tal motivo se denomina esta componente como «Factor Procesos».

Componente 4. Cinco variables componen este factor, las variables que engloba son: visión, valores, objetivos, planeación y política, de ahí que se denomine «Factor Proyección».

Componente 5. Las variables que conforman esta componente son: técnicas estadísticas, costos, medio ambiente e indicadores. Este componente agrupa variables que evidentemente están relacionadas con el control de los procesos de ahí que se denomine «Factor Control».

Componente 6: Está compuesto por las variables: perfeccionamiento, acciones preventivas, retroalimentación y comunicación. Estas variables son los pilares de la mejora continua, de ahí que se nombre a esta componente como «Factor Mejora».

Componente 7. Este es el último componente y en él se encuentran agrupadas las variables: seguridad e higiene, sistema informativo y tecnología. Teniendo en cuenta las funciones de estas variables dentro de la gestión de la calidad, se denominará «Factor Apoyo».

Con los análisis realizados se redujo el número de variables iniciales y se agruparon en 7 factores o componentes principales, quedando determinadas de esta forma, las variables que inciden en la gestión de la calidad del servicio de recepción de turismo de cruceros en el destino Santiago de Cuba.

La utilidad teórica y práctica del estudio realizado consistió en que la determinación de las variables anteriormente citadas sirvió de base para el diseño de una metodología para la gestión de la calidad del servicio de recepción del turismo de cruceros en el destino de Santiago de Cuba.

Objetivo de la metodología: Gestionar la calidad del servicio de recepción de turismo de cruceros en Santiago de Cuba, coadyuvando al proceso de toma de decisiones y mejora de la satisfacción de los cruceristas en ese destino.

En la Tabla 11 se muestra el diseño de la metodología. La misma está compuesta por seis fases que refieren etapas, herramientas y software que permiten su operacionalización. 


\section{Tabla 11 \\ DISEÑO DE LA METODOLOGÍA PARA LA GESTIÓN DE LA CALIDAD DEL SERVICIO DE RECEPCIÓN AL TURISMO \\ DE CRUCEROS EN SANTIAGO DE CUBA}

\begin{tabular}{|c|c|c|}
\hline Fases de la metodología & Herramientas seleccionadas & $\begin{array}{c}\text { Software de } \\
\text { apoyo }\end{array}$ \\
\hline \multicolumn{3}{|c|}{ Fase I: Análisis de la estrategia de desarrollo del destino Santiago de Cuba } \\
\hline \multicolumn{3}{|c|}{ Fase II: Diagnóstico del servicio de recepción de cruceros } \\
\hline $\begin{array}{l}\text { Etapa 1. Análisis de los factores externos } \\
\text { Paso 1. Análisis del macroentorno } \\
\text { Paso 2. Análisis de clientes. (TT.OO y } \\
\text { Cruceristas) } \\
\text { Paso 3. Análisis de los competidores } \\
\text { Etapa 2. Análisis de los factores internos } \\
\text { Paso 4. Análisis económico } \\
\text { Paso 5. Análisis de la oferta } \\
\text { Paso 6. Análisis de los procesos } \\
\text { Paso 7. Análisis de los proveedores de } \\
\text { servicios }\end{array}$ & $\begin{array}{l}\text { Análisis de mercado } \\
\text { Método de Pareto } \\
\text { Cuotas de mercado } \\
\text { Método del Punto Ideal } \\
\text { Alisamiento exponencial, Holt- } \\
\text { Winters } \\
\text { Valor del cliente } \\
\text { Matriz Atractividad/ } \\
\text { Competitividad } \\
\text { Matriz BCG } \\
\text { Análisis de precios } \\
\text { Matriz Fallas-Procesos } \\
\text { Método Delphi } \\
\text { Análisis de satisfacción del cliente } \\
\text { Matriz Satisfacción/Importancia } \\
\text { Método del desempeño evaluado } \\
\text { Método Valper } \\
\text { Lista de chequeo }\end{array}$ & $\begin{array}{l}\text { SPSS V } 19.0 \\
\text { Decisión } \\
\text { Microsoft Excel } \\
2010\end{array}$ \\
\hline \multicolumn{3}{|c|}{ Etapa 3. Fuerzas actuantes en la recepción de cruceros } \\
\hline \multicolumn{3}{|l|}{ Fase III: Proyección } \\
\hline Etapa 4. Análisis estructural & \multirow{4}{*}{$\begin{array}{l}\text { Matriz de Impactos Cruzados } \\
\text { Metodología Aplicada a una } \\
\text { Clasificación } \\
\text { Matriz de Alianzas, Conflictos, } \\
\text { Tácticas, Objetivos y } \\
\text { Recomendaciones } \\
\text { Sistema y Matrices de Impactos } \\
\text { Cruzados } \\
\text { Cuadro de Mando Integral }\end{array}$} & \multirow{4}{*}{$\begin{array}{l}\text { MICMAC } \\
\text { MACTOR } \\
\text { SMIC-PROB- } \\
\text { EXPERT }\end{array}$} \\
\hline Etapa 5. Análisis de los actores. & & \\
\hline Etapa 6. Definición de escenarios & & \\
\hline Etapa 7. Formulación de objetivos & & \\
\hline \multicolumn{3}{|c|}{ Fase: IV. Ejecución de la operación de recepción y atención a cruceros } \\
\hline \multicolumn{3}{|l|}{ Fase V: Control } \\
\hline $\begin{array}{l}\text { Etapa 8. Sistema de indicadores de } \\
\text { calidad para el servicio de atención al } \\
\text { turismo de crucero }\end{array}$ & \multirow[t]{2}{*}{$\begin{array}{l}\text { Dimensión social, económica, de } \\
\text { cliente, medioambiental, procesos } \\
\text { y de capacidad. }\end{array}$} & \multirow[t]{2}{*}{$\begin{array}{l}\text { Microsoft Excel } \\
2010\end{array}$} \\
\hline Etapa 9. Evaluación Integral & & \\
\hline
\end{tabular}




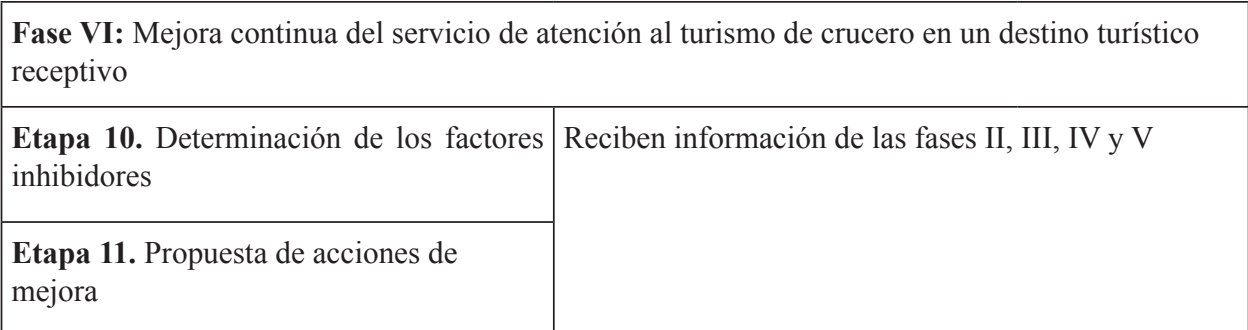

Fuente: Elaboración propia.

\section{CONCLUSIONES}

La gestión de la calidad en los servicios, posee una amplia base teórica-conceptual y continúa enriqueciéndose a través del tiempo. Sin embargo, en relación con los destinos receptivos de cruceros y con el servicio propiamente de recepción de cruceros, se han encontrado escasos trabajos, los existentes destinados, básicamente, al proceso de comercialización de esta modalidad turística. Asimismo, no se encontraron precedentes metodológicos que permitieran en el contexto cubano, la gestión de la calidad del servicio de recepción de turismo de cruceros, por lo que el objetivo tratado en este artículo científico refiere gran actualidad y pertinencia, en el plano teórico-metodológico y práctico.

El cálculo del coeficiente Alpha de Cronbach evaluó la confiabilidad y consistencia del instrumento utilizado, a su vez, permitió la exclusión de algunas variables, en las que, además de presentar incidencia nula y baja, el criterio de los expertos no fue consistente, como son, auditoría, aseguramiento metrológico, patentes, registros de calidad y servicios de postventa e inspección.

Se determinaron 33 variables que son necesarias para el análisis de la gestión de la calidad del servicio de recepción de turismo de cruceros. Resultando necesaria su consideración.

El Análisis de Componentes Principales, resultó útil para la reducción de la dimensión del conjunto de variables a considerar, demostrando que, para la gestión de la calidad del servicio de recepción de cruceros es necesaria, una adecuada combinación de siete factores o componentes, en los que se agrupan las variables que inciden en la gestión de la calidad de este servicio.

También fueron definidos factores asociados a elementos básicos de gestión de los destinos turísticos, la integración y colaboración entre los actores del destino para prestar los servicios a los cruceristas, la gestión por procesos, la proyección estratégica, el control, mejora continua y apoyo a la realización del servicio.

La determinación de los 7 factores y las variables que los componen, permitió el diseño y concepción de una metodología para la gestión de la calidad en el destino receptivo de cruceros de Santiago de Cuba, susceptible de ser generalizada a otros destinos turísticos del país. La misma será publicada por los autores de este artículo como contribución a la mejora de la calidad y el proceso de toma de decisiones en los destinos turísticos receptivos de cruceros. 


\section{BIBLIOGRAFÍA}

1. AYALA CASTRO, H. (2011): «Una mirada a 10 años de turismo en el Caribe Insular», Turismo y Desarrollo Local, vol. 4, $\mathrm{n}^{\circ}$ 11, pp. 1-27.

2. BARBOSA, A. J., \& GUTIÉRREZ, O. P. (2010): «Propuesta de un procedimiento para el análisis de la demanda turística». Turismo y Desarrollo Local, vol. 3, n 7, pp. 1-15.

3. BARÓMETRO OMT. ORGANIZACIÓN MUNDIAL DEL TURISMO (2013): Anexo estadístico. Organización Mundial del Turismo, Madrid, España, vol. 11.

4. CRONIN, J.J. y TAYLOR, S.A. (1994): «SERVPERF vs SERVQUAL: Reconciling PerformanceBased and Perceptions-Minus-Expectations Measurement of Service Quality». Journal of Marketing, vol. 58, pp. 15-131.

5. CRUISE LINES INTERNATIONAL ASSOCIATION. (2014): Cruise Market Overview: Statistical Cruise Industry. Data through 2013. Accessed January, 25, 2015.

6. CRUISE INDUSTRY NEWS ANNUAL. (2013): International Guide to Cruise Industry, Cruise Industry News, New York, NY. USA.

7. DACHARY, A.C. y ARNAIZ BURNE, S.M. (2006): «El estudio del turismo: ¿Un paradigma en formación?». Estudios y Perspectivas en Turismo, vol. 15, n², pp. 179192.

8. FERNÁNDEZ, J.I.P. y RIVERO, M.S. (2010): «Competitividad versus crecimiento en destinos turísticos. Un análisis mediante técnicas multivariantes», Cuadernos de Economía, vol. 33, n 91, pp. 159-181.

9. FERREIRA LOPES, S. D. y VELOSO DA SILVA, J.A. (2011): «Análisis estratégico de la oferta turística portuguesa: El análisis de importancia-valoración (IPA)», Estudios y Perspectivas en Turismo, vol. 20, n 5, pp. 997-1.008.

10. FIGUEROLA, M. (1999): Introducción al Estudio Económico del Turismo. Civitas. Madrid.

11. FLORIDA-CARIBBEAN CRUISE ASSOCIATION (2014): Excerpts from the CHTA Caribbean Travel Marketplace's Official Opening. Portia Simpson Miller. Prime Minister of Jamaica.

12. GETTY, J. y THOMPSON, K. (1994): «A procedure for scaling perceptions of lodging quality», Hospitality Research Journal, vol. 18, n², pp. 75-96.

13. HAIR, J. F., SARSTEDT, M., RINGLE, C. M. y MENA, J.A. (2012): «An assessment of the use of partial least squares structural equation modeling in marketing research», Journal of the Academy of Marketing Science, vol. 40, n 3, pp. 414-433.

14. JÖNSSON, C. y DEVONISH, D. (2009): «An exploratory study of competitive strategies among hotels in a small developing Caribbean state», International Journal of Contemporary Hospitality Management, vol. 21, $\mathrm{n}^{0}$ 4, pp. 491-500.

15. LIZASOAIN, L y JOARISTI. L. (2003): Gestión y análisis de datos con SPSS versión 11. Universidad del País Vasco. Thomson-Paraninfo. Madrid.

16. MARTÍNEZ, E.O., HERRÁEZ, B.R. y MANGIN, J.P.L. (2008): « Percepción de los servicios de apoyo en el sector hotelero. Un análisis internacional», Cuadernos de Turismo, $\mathrm{n}^{0} 21$, pp. 113-134.

17. MORA CONTRERAS, C.E. (2011): «La Calidad del Servicio y la Satisfacción del Consumidor», Revista Brasileira de Marketing, vol. 10, n² 2, pp. 146-162. 
18. MOCK DÍAZ JOAQUÍN. (2007): Modelo de Excelencia para la Agencia de Viajes Cubanacan Santiago de Cuba. Tesis Doctoral. Universidad de Oriente. Cuba.

19. MINISTERIO DE TURISMO (2013): Plan estratégico de desarrollo del destino turístico de Santiago de Cuba, 2013. Cuba, 54 pp.

20. PREMIO NACIONAL DE CALIDAD DE LA REPÚBLICA DE CUBA. (2013): Bases y Guía de Autoevaluación. Cuba. 121 pp.

21. ROBLES, C.F. y LÓPEZ, A.M.G. (2009): «La apuesta por la calidad como elemento diferenciador en los destinos turísticos: planes renovados», Cuadernos de Turismo, $\mathrm{n}^{0}$ 23, pp. 89-110.

22.SÁNCHEZ MENDOZA, V.V. (2012): «La percepción de los cruceristas estadounidenses sobre el servicio turístico de calidad ofrecido por los guías de turistas en Mazatlán, México», Anuario Turismo y Sociedad, n 13, pp. 185-208.

23.SANDERS, E. y LONG, P. (2002): «Economic Benefits to the United States from Lifting the Ban on Travel to Cuba». The Cuban Policy Foundation Washington, DC.

24. VERGARA, J. C., QUESADA, V. M. y BLANCO, I. (2011):«Análisis de la calidad en el servicio y satisfacción de los usuarios en dos hoteles cinco estrellas de la ciudad de Cartagena (Colombia) mediante un modelo de ecuaciones estructurales», Ingeniare. Revista chilena de ingeniería, vol. 19, n 3, pp. 420-428.

25. VOGEL, M. (2011): «Monopolies at Sea: The Role of Onboard Sales for the Cruise Industry's Growth and Profitability», Tourism Economics, Physica-Verlag HD, pp. 211-229. 


\section{ANEXO 1}

\section{a. Relación de variables presentes en los modelos analizados}

\begin{tabular}{|c|c|c|c|c|c|c|c|c|c|c|c|c|}
\hline No & Variables & 1 & 2 & 3 & 4 & 5 & 6 & 7 & 8 & 9 & 10 & 11 \\
\hline 1 & Acciones preventivas & & $\mathrm{x}$ & & & & $\mathrm{x}$ & & & $\mathrm{x}$ & $\mathrm{x}$ & $\mathrm{x}$ \\
\hline 2 & Auditoria & & & & $\mathrm{x}$ & & $\mathrm{x}$ & & & $\mathrm{x}$ & & $\mathrm{x}$ \\
\hline 3 & Aseguramiento metrológico & & & & & & & & & & $\mathrm{x}$ & $\mathrm{x}$ \\
\hline 4 & Cliente & $\mathrm{x}$ & $\mathrm{x}$ & $\mathrm{x}$ & $\mathrm{x}$ & $\mathrm{x}$ & $\mathrm{x}$ & $\mathrm{x}$ & $\mathrm{x}$ & $\mathrm{x}$ & $\mathrm{x}$ & $\mathrm{x}$ \\
\hline 5 & Comunicación & $\mathrm{x}$ & & $\mathrm{x}$ & & & $\mathrm{x}$ & & $\mathrm{x}$ & $\mathrm{x}$ & $\mathrm{x}$ & $\mathrm{x}$ \\
\hline 6 & Indicadores & & & & & & & & & $\mathrm{x}$ & & $\mathrm{x}$ \\
\hline 7 & Costo & & & & & & & $\mathrm{x}$ & $\mathrm{x}$ & $\mathrm{x}$ & & $\mathrm{x}$ \\
\hline 8 & Documentos & & & $\mathrm{x}$ & & & & $\mathrm{x}$ & & & & $\mathrm{x}$ \\
\hline 9 & Actividades-operaciones & $\mathrm{x}$ & $\mathrm{x}$ & & $\mathrm{x}$ & & $\mathrm{x}$ & $\mathrm{x}$ & $\mathrm{x}$ & $\mathrm{x}$ & $\mathrm{x}$ & $\mathrm{x}$ \\
\hline 10 & Inspección & & $\mathrm{x}$ & & $\mathrm{x}$ & & & $\mathrm{x}$ & & & $\mathrm{x}$ & \\
\hline 11 & Liderazgo & & & & & & & & & $\mathrm{x}$ & $\mathrm{x}$ & $\mathrm{x}$ \\
\hline 12 & Medio ambiente & & & & $\mathrm{x}$ & & & & & $\mathrm{x}$ & $\mathrm{x}$ & $\mathrm{x}$ \\
\hline 13 & Perfeccionamiento & $\mathrm{x}$ & & & & & $\mathrm{x}$ & & $\mathrm{x}$ & & & \\
\hline 14 & Comercialización & & $\mathrm{x}$ & & & & & & $\mathrm{x}$ & $\mathrm{x}$ & $\mathrm{x}$ & $\mathrm{x}$ \\
\hline 15 & Motivación & & & & & $\mathrm{x}$ & & & & & $\mathrm{x}$ & \\
\hline 16 & Objetivos & & $\mathrm{x}$ & & $\mathrm{x}$ & & & $\mathrm{x}$ & & $\mathrm{x}$ & $\mathrm{x}$ & $\mathrm{x}$ \\
\hline 17 & Organización & $\mathrm{x}$ & $\mathrm{x}$ & & & $\mathrm{x}$ & $\mathrm{x}$ & & $\mathrm{x}$ & $\mathrm{x}$ & $\mathrm{x}$ & $\mathrm{x}$ \\
\hline 18 & Patentes & & & & & & & & & $\mathrm{x}$ & $\mathrm{x}$ & \\
\hline 19 & Planeación & & & & & & & & & $\mathrm{x}$ & $\mathrm{x}$ & $\mathrm{x}$ \\
\hline 20 & Política & & $\mathrm{x}$ & & $\mathrm{x}$ & & & $\mathrm{x}$ & $\mathrm{x}$ & $\mathrm{x}$ & & $\mathrm{X}$ \\
\hline 21 & Personal & $\mathrm{x}$ & & & & & $\mathrm{x}$ & & & $\mathrm{x}$ & $\mathrm{x}$ & $\mathrm{x}$ \\
\hline 22 & Principios & & & $\mathrm{x}$ & & $\mathrm{x}$ & & & & & $\mathrm{x}$ & $\mathrm{X}$ \\
\hline 23 & Producto o Servicio & $\mathrm{x}$ & $\mathrm{x}$ & $\mathrm{x}$ & $\mathrm{x}$ & $\mathrm{x}$ & $\mathrm{x}$ & $\mathrm{x}$ & $\mathrm{x}$ & $\mathrm{x}$ & $\mathrm{x}$ & $\mathrm{x}$ \\
\hline 24 & Recursos materiales & & $\mathrm{x}$ & $\mathrm{x}$ & & & & & & $\mathrm{x}$ & $\mathrm{x}$ & $\mathrm{x}$ \\
\hline 25 & Registros de calidad & $\mathrm{x}$ & & $\mathrm{x}$ & $\mathrm{X}$ & $\mathrm{X}$ & $\mathrm{X}$ & & & $\mathrm{x}$ & & \\
\hline 26 & $\begin{array}{l}\text { Proveedores de servicios } \\
\text { turísticos }\end{array}$ & & & & & & & & & & $\mathrm{X}$ & $\mathrm{X}$ \\
\hline 27 & Selección & $\mathrm{x}$ & & & & & & & & $\mathrm{x}$ & & $\mathrm{X}$ \\
\hline
\end{tabular}




\begin{tabular}{|l|l|c|c|c|c|c|c|c|c|c|c|c|}
\hline $\mathbf{2 8}$ & Servicios de postventa & $\mathrm{x}$ & & $\mathrm{x}$ & & & $\mathrm{x}$ & & & & & $\mathrm{x}$ \\
\hline $\mathbf{2 9}$ & Seguridad e higiene & & & & & & & & & $\mathrm{x}$ & & $\mathrm{x}$ \\
\hline $\mathbf{3 0}$ & Sistema informativo & & & & & & & $\mathrm{x}$ & & $\mathrm{x}$ & $\mathrm{x}$ & $\mathrm{x}$ \\
\hline $\mathbf{3 1}$ & Sociedad & & & & $\mathrm{x}$ & & & & & $\mathrm{x}$ & $\mathrm{x}$ & $\mathrm{x}$ \\
\hline $\mathbf{3 2}$ & Tecnología & $\mathrm{x}$ & $\mathrm{x}$ & & & $\mathrm{x}$ & $\mathrm{x}$ & & & & $\mathrm{x}$ & $\mathrm{x}$ \\
\hline $\mathbf{3 3}$ & Técnicas estadísticas & & & & & $\mathrm{x}$ & & & & $\mathrm{x}$ & $\mathrm{x}$ & $\mathrm{x}$ \\
\hline $\mathbf{3 4}$ & Valores & & & & & & & & & $\mathrm{x}$ & $\mathrm{x}$ & $\mathrm{x}$ \\
\hline $\mathbf{3 5}$ & Visión & & $\mathrm{x}$ & & & & & & & $\mathrm{x}$ & $\mathrm{x}$ & $\mathrm{x}$ \\
\hline $\mathbf{3 6}$ & Retroalimentación & & & $\mathrm{x}$ & $\mathrm{x}$ & & & & & & $\mathrm{x}$ & $\mathrm{x}$ \\
\hline $\mathbf{3 7}$ & Implicados en el servicio & & & & $\mathrm{x}$ & & & & $\mathrm{x}$ & $\mathrm{x}$ & $\mathrm{x}$ & \\
\hline $\mathbf{3 8}$ & Fidelización & $\mathrm{x}$ & $\mathrm{x}$ & $\mathrm{x}$ & $\mathrm{x}$ & $\mathrm{x}$ & $\mathrm{x}$ & $\mathrm{x}$ & $\mathrm{x}$ & $\mathrm{x}$ & $\mathrm{x}$ & $\mathrm{x}$ \\
\hline $\mathbf{3 9}$ & Satisfacción & & & \\
\hline
\end{tabular}

Leyenda: 1. Modelo SERVQUAL, 2. Modelo SERVPERF, 3. Modelo de Teas, 4. Modelo de Fishbein, 5. Modelo de Calidad de Grouroos - Gummenson, 6. Modelo Servman, 7. Modelo LODGQUAL, 8. Modelo Valper, 9. Sistema Integral de Calidad Turística en Destinos (SICTED), 10. Premio Nacional de Calidad de la República de Cuba, 11. Modelo de Excelencia para la Agencia de Viajes Cubanacan Santiago.

\section{b. Listado de variables identificadas en los modelos analizados}

\begin{tabular}{|c|l|l|}
\hline No & Listado de Variables & $\begin{array}{l}\text { Breve significado de las variables en el contexto de } \\
\text { la investigación }\end{array}$ \\
\hline 1 & Acciones preventivas & $\begin{array}{l}\text { Las acciones preventivas se anticipan a la causa de } \\
\text { insatisfacciones, y pretenden eliminarla o atenuarlas } \\
\text { antes de su existencia. Evitan los problemas identifi- } \\
\text { cando los riesgos. }\end{array}$ \\
\hline 2 & Auditoria & $\begin{array}{l}\text { Examen físico y documental del sistema de gestión } \\
\text { para la atención a los cruceristas. }\end{array}$ \\
\hline 3 & Aseguramiento metrológico & $\begin{array}{l}\text { Acciones que mantienen y controlan el correcto fun- } \\
\text { cionamiento del sistema mediante instrumentos de } \\
\text { medición e indicadores del sistema de gestión. }\end{array}$ \\
\hline 4 & Cliente & Usuarios del sistema, cruceristas. \\
\hline 5 & Comunicación & $\begin{array}{l}\text { Proceso mediante el cual se transmite la información } \\
\text { precisa para el funcionamiento del sistema de atención } \\
\text { a los cruceristas. }\end{array}$ \\
\hline 6 & Indicadores & $\begin{array}{l}\text { Elementos que se utilizan para indicar o señalar al- } \\
\text { gún resultado asociado a la gestión de cruceros en el } \\
\text { destino turístico }\end{array}$ \\
\hline
\end{tabular}




\begin{tabular}{|c|c|c|}
\hline 7 & Costo & $\begin{array}{l}\text { Expresión monetaria de los consumos de los factores } \\
\text { utilizados en el servicio de atención a cruceros en el } \\
\text { destino turístico. }\end{array}$ \\
\hline 8 & Documentos & $\begin{array}{l}\text { Evidencia, testimonio material de todas las opera- } \\
\text { ciones realizadas en los procesos de atención a los } \\
\text { cruceros en el destino turístico. }\end{array}$ \\
\hline 9 & Actividades-operaciones & $\begin{array}{l}\text { Instrucciones de trabajo para realizar tareas muy con- } \\
\text { cretas en los procesos turísticos relacionados con el ser- } \\
\text { vicio de atención a los cruceristas en el destino turístico. }\end{array}$ \\
\hline 10 & Inspección & $\begin{array}{l}\text { Examen y revisión física del cumplimiento de están- } \\
\text { dares de calidad del servicio de atención a cruceros. }\end{array}$ \\
\hline 11 & Liderazgo & $\begin{array}{l}\text { Habilidades gerenciales o directivas que distinguen a } \\
\text { organizaciones y personas en la gestión de la calidad } \\
\text { del destino turístico. }\end{array}$ \\
\hline 12 & Medio ambiente & $\begin{array}{l}\text { Conjuntos de componentes físicos, químicos, bioló- } \\
\text { gicos, sociales, económicos y culturales que influyen } \\
\text { directamente sobre la calidad del servicio de los cru- } \\
\text { ceristas, atractivos y accesos del destino turístico. }\end{array}$ \\
\hline 13 & Perfeccionamiento & $\begin{array}{l}\text { Mejora continua en los procesos orientados a la satis- } \\
\text { facción de los turistas. }\end{array}$ \\
\hline 14 & Comercialización & $\begin{array}{l}\text { Proceso que comprende la venta de actitudes, bene- } \\
\text { ficios y/o satisfacciones, es decir, servicios materia- } \\
\text { lizadas en excursiones turísticas del destino turístico } \\
\text { receptivo. }\end{array}$ \\
\hline 15 & Motivación & $\begin{array}{l}\text { Conjunto de estímulos e incentivos que mueven a los } \\
\text { empleados y gerentes de organizaciones implicadas } \\
\text { en el servicio de atención a los cruceristas para ofre- } \\
\text { cer un servicio de calidad, cumplir los estándares y } \\
\text { superar las expectativas }\end{array}$ \\
\hline 16 & Objetivos & $\begin{array}{l}\text { La variable mide el grado en que la misión y objeti- } \\
\text { vos estratégicos están enfocados al logro de la cali- } \\
\text { dad del servicio en la atención al turismo de cruceros. }\end{array}$ \\
\hline 17 & Organización & $\begin{array}{l}\text { Nivel de comunicación y acción coordinada en la } \\
\text { gestión de la calidad del destino turístico. }\end{array}$ \\
\hline 18 & Patentes & $\begin{array}{l}\text { La existencia de registros, marcas en el destino tu- } \\
\text { rístico y el grado en que influyen en la calidad de } \\
\text { atención al servicio de cruceros. }\end{array}$ \\
\hline 19 & Planeación & $\begin{array}{l}\text { Comprende el análisis de una situación, el estableci- } \\
\text { miento de objetivos, la formulación de estrategias y } \\
\text { planes de acción. En el contexto de esta investigación } \\
\text { se valoró su enfoque prospectivo. }\end{array}$ \\
\hline
\end{tabular}




\begin{tabular}{|c|c|c|}
\hline 20 & Política & $\begin{array}{l}\text { Entiende la existencia y medida del impacto real de } \\
\text { las acciones concretas orientadas a la acción integra- } \\
\text { da en el destino turístico para alcanzar los objetivos } \\
\text { de calidad. }\end{array}$ \\
\hline 21 & Personal & $\begin{array}{l}\text { Grado de participación de las personas en la atención } \\
\text { al turismo de crucero y que están representadas por } \\
\text { organizaciones empresariales e instituciones en el } \\
\text { destino turístico. }\end{array}$ \\
\hline 22 & Principios & $\begin{array}{l}\text { Refiere el nivel en que los implicados en la atención a } \\
\text { los cruceros adoptan los ocho principios generalmen- } \\
\text { te aceptados en la gestión de la calidad. }\end{array}$ \\
\hline 23 & Producto o Servicio & $\begin{array}{l}\text { Mide la estabilidad y variedad de la oferta y el grado } \\
\text { en que el producto turístico concreto en su integra- } \\
\text { ción con los servicios, equipamientos e infraestruc- } \\
\text { turas permite satisfacer necesidades, motivaciones y } \\
\text { expectativas de los cruceristas en el destino turístico. }\end{array}$ \\
\hline 24 & Recursos materiales & $\begin{array}{l}\text { Refiere el estado de los bienes que forman parte del } \\
\text { proceso de atención a los cruceros en el destino. }\end{array}$ \\
\hline 25 & Registros de calidad & $\begin{array}{l}\text { Nivel en que están establecidos los medios para su- } \\
\text { ministrar pruebas de conformidad con los requisitos } \\
\text { de calidad en la atención a los cruceros en el destino } \\
\text { turístico. }\end{array}$ \\
\hline 26 & $\begin{array}{l}\text { Proveedores de servicios turísti- } \\
\cos \end{array}$ & $\begin{array}{l}\text { Refiere el grado participación de todos los actores } \\
\text { implicados en la atención al turismo de cruceros en } \\
\text { un destino receptivo. }\end{array}$ \\
\hline 27 & Selección & $\begin{array}{l}\text { Proceso dirigido a seleccionar las mejores alterna- } \\
\text { tivas para ofrecer un servicio de calidad a los cru- } \\
\text { ceristas en correspondencia a las expectativas de los } \\
\text { clientes y capacidad real del destino turístico. }\end{array}$ \\
\hline 28 & Servicios de postventa & $\begin{array}{l}\text { Servicios promocionales, de seguridad y manteni- } \\
\text { miento a los turoperadores turísticos y cruceristas en } \\
\text { el destino turístico. }\end{array}$ \\
\hline 29 & Seguridad e higiene & $\begin{array}{l}\text { Requisito de calidad valorado por los cruceristas en } \\
\text { destinos turísticos receptivos como parte de una ofer- } \\
\text { ta turística integral. }\end{array}$ \\
\hline 30 & Sistema informativo & $\begin{array}{l}\text { Refiere el conjunto de elementos orientados al tra- } \\
\text { tamiento y administración de la información para la } \\
\text { gestión de la calidad en el servicio de cruceros en el } \\
\text { destino turístico. }\end{array}$ \\
\hline 31 & Sociedad & $\begin{array}{l}\text { Integración de todos los actores en la prestación del } \\
\text { servicio de calidad a los cruceristas. Se atribuye gran } \\
\text { importancia al nivel en que lo hace la comunidad local. }\end{array}$ \\
\hline
\end{tabular}




\begin{tabular}{|c|c|c|}
\hline 32 & Tecnología & $\begin{array}{l}\text { Grado en que los conocimientos científicos y adelan- } \\
\text { tos en términos de comunicación e informática son } \\
\text { utilizados con efectividad en el servicio de atención } \\
\text { a los cruceros. }\end{array}$ \\
\hline 33 & Técnicas estadísticas & $\begin{array}{l}\text { La existencia y grado de empleo de estudios estadís- } \\
\text { ticos para estimar resultados de la gestión de calidad } \\
\text { en el destino turístico y tomar decisiones oportunas } \\
\text { y fiables. }\end{array}$ \\
\hline 34 & Valores & $\begin{array}{l}\text { Refiere el nivel de contribución de los valores com- } \\
\text { partidos por las organizaciones a favor de la calidad } \\
\text { en la atención a cruceros en el destino turístico. }\end{array}$ \\
\hline 35 & Visión & $\begin{array}{l}\text { Nivel de compromiso de las organizaciones implica- } \\
\text { das en la calidad de atención a los cruceros en cuanto } \\
\text { a las metas y mejora continua de procesos y sistemas. }\end{array}$ \\
\hline 36 & Retroalimentación & $\begin{array}{l}\text { Nivel de efectividad en que se regula el comporta- } \\
\text { miento y controla el sistema de calidad de atención al } \\
\text { turismo de cruceros en el destino turístico. }\end{array}$ \\
\hline 37 & Implicados en el servicio & $\begin{array}{l}\text { Es más que participación. Refiere el grado de actua- } \\
\text { ción de los actores locales como parte de su filosofía } \\
\text { de gestión con relación a la calidad del servicio de } \\
\text { atención a cruceros en el destino. }\end{array}$ \\
\hline 38 & Fidelización & $\begin{array}{l}\text { Grado de lealtad de los turoperadores de cruceros en } \\
\text { el destino turístico. }\end{array}$ \\
\hline 39 & Satisfacción & $\begin{array}{l}\text { Se asocia a la calidad de servicio y refiere el grado de } \\
\text { ajuste entre las expectativas y la percepción final del } \\
\text { resultado del servicio. }\end{array}$ \\
\hline
\end{tabular}


